\title{
FEMTOSECOND STUDIES OF ELECTRON PHOTODETACHMENT FROM AN IODIDE ION IN SOLUTION: THE TRAPPED ELECTRON
}

\author{
Frederick H. LONG ${ }^{1}$, Hong LU, Xuelong SHI and Kenneth B. EISENTHAL ${ }^{2}$ \\ Department of Chemistry, Columbia University, New York, NY 10027, USA
}

Received 23 February 1990; in final form 28 Mạrch 1990

\begin{abstract}
We have performed femtosecond studies of electron photodetachment from a simple halide ion in aqueous solution. After photoexcitation, the electron is trapped in the deep potential well formed by the orientational polarization of the water molecules around the anion. This trapped state of the electron is a precursor of the wet electron and in the case of an aqueous iodide ion has a strong absorption in the visible. We hypothesize that the electron recombines with the neutral halogen atom or crosses onto the potential surface of the wet electron by a non-adiabatic electron transfer process. We find that analogous to the case of photoionization of a neutral water molecule, the wet to solvated electron transition in the vicinity of the halogen atom can be described as two-state in character.
\end{abstract}

\section{Introduction}

The behavior of simple ions in aqueous solution has long been of prime interest to physical chemists and is important for understanding many chemical and biological processes. Many simple anions in solution such as halides have large, broad absorption spectra in the UV. Over thirty years ago, it was discovered that upon the absorption of light, solvated electrons were produced $[1,2]$. Some workers postulated that photoionization was occurring; however, this was an incomplete explanation. Photoionization involves a direct transition of an electron to a continuum state; photodetachment is often used to describe the analogous process for an anion [3]. In the case of many simple ions, there are good reasons to believe that the electron makes a transition to a state inside the potential well formed by the water molecules solvated around the anion [4,5]. The absorption bands that correspond to the removal of the electron from the ion are called charge transfer to solvent spectra or CTTS spectra (for a general review, see ref. [6]). CTTS transitions are unique to the condensed phase and are unrelated to the well-

\footnotetext{
IBM pre-doctoral fellow.

2 To whom correspondence should be addressed.
}

known phenomena of autoionization and Rydberg states which are not due to environmental effects [3].

Little work has been done on the dynamics of the CTTS transitions and the subsequent solvation dynamics of the electron following photodetachment [7]. We have done a detailed study, with improved time resolution, of the electron photodetachment dynamics in a series of aqueous halides. In this Letter, we will discuss our experiments on the aqueous iodide ion $\mathrm{I}^{-}$, and how they relate to the CTTS dynamics. The solvation dynamics of the electron produced are very different than in the neat water case where the electron is produced by photoionization $[7,8]$. In this case the precursor of the wet electron is a continuum state called the quasi-free electron. For the halide anion, the precursor of the wet electron is a bound state in the CTTS well, the trapped electron. The very early time dynamics of the electron are strongly influenced by these short range molecular effects.

\section{Experimental}

The experiments reported here were performed using an amplified colliding pulse mode-locked dye laser operating at $10 \mathrm{~Hz}, 625 \mathrm{~nm}, 80 \mathrm{fs}$ fwhm and 
$300 \mu \mathrm{J} /$ pulse. The $312.5 \mathrm{~nm}(3.94 \mathrm{eV})$ pump beam was generated by frequency doubling the CPM beam with a $200 \mu \mathrm{m}$ BBO crystal. The photodetachment threshold potential of the aqueous iodide is $5.5 \mathrm{eV}$ [6]; therefore a two-photon excitation is needed. Care was taken to defocus the pump light in order to avoid photoionizing the solvent. The probe beam was either a small fraction of the fundamental beam or part of a continuum pulse generated in a $1 \mathrm{~cm}$ pathlength cell of $\mathrm{D}_{2} \mathrm{O}$.

The water used was HPLC grade and the Nal was the highest grade purity form Aldrich. Experiments with KI yielded identical results. The concentration of $\mathrm{KI}$ and $\mathrm{NaI}$ used was $1 \mathrm{M}$. At this concentration, departures from the dielectric and transport properties of neat water are less than $10 \% \# 1$.

\section{Background}

One of the first and most successful models of the CTTS absorption spectra was proposed by Platzman and Frank [10]. At this point in time, it was known that upon the absorption of light, an electron could be detached from an aqueous ion,

$\mathrm{X}^{-}+h \boldsymbol{\nu} \rightarrow \mathrm{X}+\mathrm{e}_{\mathrm{aq}}^{-}$.

According to the Franck-Condon principle, the molecules surrounding the solvated ion cannot move on the time scale of the optical absorption. Therefore the excited electron will only experience a potential field due to the orientational polarization of the water molecules, which cannot change on the time scale of absorption. Therefore, the total potential energy of an electron in this well is

$U_{\text {pol }}=U_{\text {total }}-U_{\text {elec }}$,

$U(r)=-\frac{e^{2}}{r}\left(\frac{1}{\epsilon_{\infty}}-\frac{1}{\epsilon_{0}}\right)$,

where $U_{\text {total }}$ is the equilibrium potential energy, $U_{\text {elec }}$ is the interaction energy due to electronic polarization, $U_{\mathrm{pol}}$ is due to the orientational polarization; $\epsilon_{0}$ is the static dielectric constant and $\epsilon_{\infty}$ is the high frequency dielectric constant. This is the famous result

\#1 Using the known dielectric properties of a $1 \mathrm{M} \mathrm{NaCl}$ solution, a $7 \%$ increase in the longitudinal response time is expected [9]. of Landau's theory of polarons, i.e. the self-trapping of an electron in an ionic lattice [11]. $U_{\text {pol }}$ can be interpreted as the potential well "dug" by the electron after it is placed in the polar liquid. It is important to note that since the static dielectric constant of water is large (about 80 ), only the high frequency dielectric constant enters into eq. (3). Physically this means that the potential felt by the electron is quite strong compared to that felt in the equilibrium situation. A simple calculation shows that this potential is on the order of 2-3 eV deep.

Frank and Platzman used the above result from Landau's theory of polarons to help predict the CTTS transition maxima for the aqueous halide ions. An improved version of this theory was developed by Stein and Treinin [12]. Both theories construct the CTTS transition energy from a thermodynamic cycle. The most important components of the CTTS transition energy are the gas phase electron detachment energy (electron affinity) and the binding energy of the electron to the polarization well of the oriented water molecules. Using Landau's polaron model for the electron binding energy in the well, i.e. eq. (3), qualitative agreement could be obtained between the halide spectra and theory. An alternative to the Frank-Platzman model which was developed by Smith and Symons views the polarization as an infinite square well potential [13]. It is important to note that both models predict that the CTTS threshold energies and the excited state energy level separations increase as the halide ion radius decreases. This can most simply be seen by noting that the energy separation in an infinite well scales as $1 / L^{2}$, where $L$ is the box length. Clearly the situation is more complex than portrayed in these simple macroscopic models; however, as is often the case simple models can be used to predict qualitative trends.

\section{Results and discussion}

In figs. 1-3, the observed dynamics of the aqueous electron originating from the iodide ion are shown. Fig. 1, data taken with a $1000 \mathrm{~nm}$ IR probe pulse is qualitatively similar but quantitatively different than the neat water case. After a delayed appearance, the absorption increases then slowly decays. The rise of the signal corresponds to the formation of the wet 


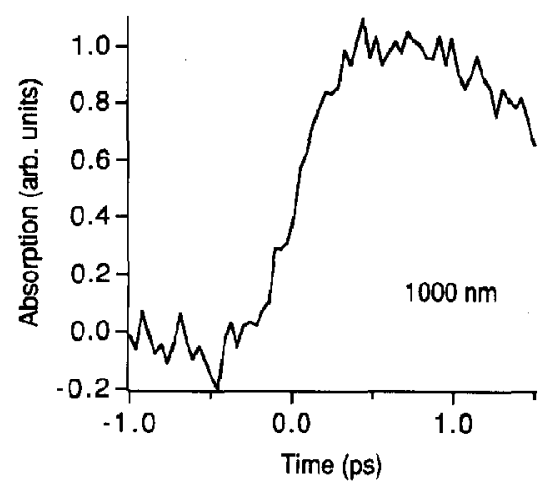

Fig. 1. Dynamics of the photodetached electron probed at 1000 $\mathrm{nm}$.

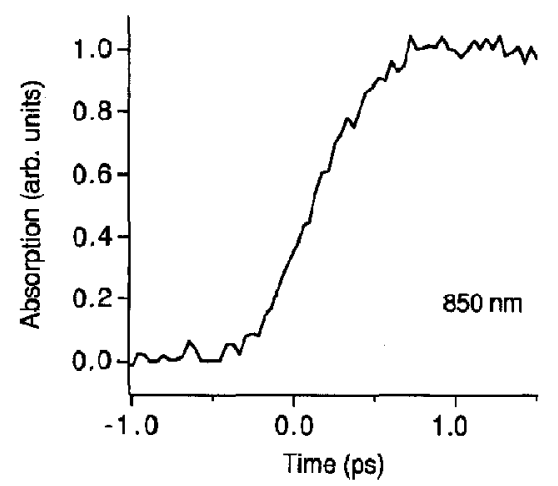

Fig. 2. Dynamics of the photodetached electron probed at 850 $\mathrm{nm}$. The appearance of an isosbestic point is evident.

electron and the decay to the formation of the fully solvated electron. In fig. 2 the photodetachment dynamics are probed at $850 \mathrm{~nm}$. After the formation of the wet electron, the dynamics appear to be constant. This is the signature of the existence of an isosbestic point in the dynamics. In fig. 3 a $625 \mathrm{~nm}$ probe was used and a dramatic departure from previous experiments is observed. A large rise and decay at early times is present in the data. The signal appears to be a precursor of the wet electron. We believe this is due to the absorption of the electron before it has left the potential well around the once solvated ion, i.e. absorption from the photoexcited CTTS state. This will be discussed in more detail below.

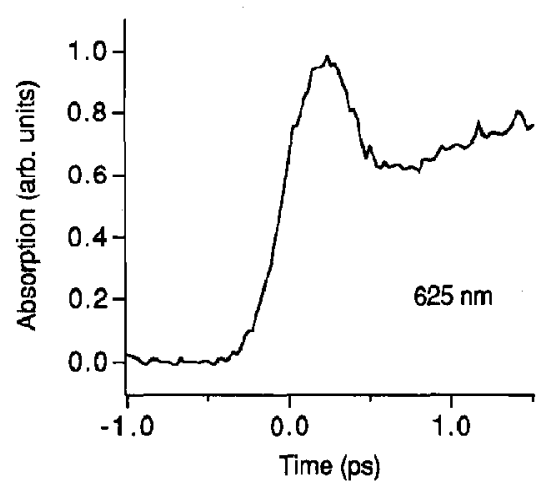

Fig. 3. Dynamics of the photodetached electron probed at 625 $\mathrm{nm}$. The appearance of an precursor species of the wet electron is quite evident.

\subsection{Dynamics of the wet electron}

The dynamics of electrons in liquid water have been of great interest theoretically [14,15] (for an extensive review of the literature, see ref. [16]) and experimentally $[8,17-20]$ in recent years. Our most recent experiments on electron solvation in liquid water have been recently reported [8]. Since they are essential in understanding the experiments discussed here, we will summarize them. The formation time, decay time and absorption spectra of the wet or pre-solvated electron were measured. An isosbestic point, a wavelength where the absorption coefficient of the two states are equivalent, was found in the transition of the wet to the solvated electron. At the isosbestic point, the absorption signal was constant after the formation of the wet electron; this is strong experimental evidence for the two-state model. In other words the transition of the wet electron into the solvated electron is not continuous in character, i.e. there is not a continuous sequence of wet electrons leading to a solvated electron. We have proposed that the wet electron is simply the excited state of the solvated electron and that the rate limiting step in the solvation dynamics is really an internal conversion process [8]. This is consistent with the nonadiabatic process proposed by Rossky and Schnitker [14] to explain the difference in the observed solvation dynamics and calculations of adiabatic solvation dynamics.

The first step in the analysis of the data is the de- 
termination of the isosbestic point if it exists. We located an isosbestic point to be at $850 \mathrm{~nm}$. This is very close to the $820 \mathrm{~nm}$ obtained for the neat water. From a single exponential fit, the appearance time of the wet electron was found to be $300 \pm 50 \mathrm{fs}$. This is about $50 \%$ longer than the time found for neat water [8]. However most importantly, the determination of the isosbestic point shows that the wet electron to solvated electron transition is describable as a two-state process. Therefore, our interpretation of the wet electron in neat water as an excited state of the solvated electron is probably still valid in the case of photodetachment from simple ions via a CTTS state. Furthermore this result suggests that it is appropriate to use a kinetic model to analyze the data. In contrast to the neat water case, the wet electron precursor can be seen directly; therefore, the model must include the kinetics of this species, the trapped electron, i.e. the electron in the very short lived CTTS state,

$\mathrm{e}_{\text {trapped }} \stackrel{k_{1}}{\longrightarrow} \mathrm{e}_{\text {wet }} \stackrel{k_{2}}{\longrightarrow} \mathrm{e}_{\text {solvated }}$

By solving the appropriate rate equations one obtains

$$
\begin{aligned}
& N(t)=N_{0}\left\{\left[\frac{k_{1}}{k_{1}-k_{2}}\left(\epsilon_{\mathrm{wet}}-\epsilon_{\mathrm{sol}}\right) \exp \left(-k_{2} t\right)\right.\right. \\
& \left.+\epsilon_{\mathrm{sol}}+\left(\frac{k_{2} \epsilon_{\mathrm{sol}}-k_{1} \epsilon_{\mathrm{wet}}}{k_{1}-k_{2}}\right) \exp \left(-k_{1} t\right)\right] \\
& \left.+\epsilon_{\mathrm{CTTS}} \exp \left(-k_{1} t\right)\right\} .
\end{aligned}
$$

$N_{0}$ is the number of electrons produced by the excitation pulse; $k_{1}$ is the radiationless decay rate of the trapped electron; $k_{2}$ is the formation rate of the solvated electron; $\epsilon_{\mathrm{CTT}}, \epsilon_{\text {wet }}$ and $\epsilon_{\mathrm{sol}}$ are the absorption coefficients of the CTTS, wet and solvated electrons, respectively. The data at all wavelengths were analyzed using this model. It is well established that the absorption spectra of the solvated electron is unaffected by the presence of ions in $1 \mathrm{M}$ concentrations. Therefore as we did in the neat water experiments we can find the wet electron absorption spectra in the iodide solution, by comparison with the known solvated electron absorption. At much higher concentrations the absorption spectra of the solvated electron will be a function of ion concentration [21].
The wet electron absorption spectra found from our experiments on $\mathrm{I}^{-}$is quite similar to the wet electron photoionized from a neutral water molecule. The decay time of the wet electron, $1 / k_{2}$, extracted from the fit was the same as neat water $540 \pm 50 \mathrm{fs}$. This result and the similarity of the wet electron absorption spectra to that obtained for neat water suggest that the largest deviations from the neat water case occur at early times.

\subsection{The precursor of the wet electron: a trapped electron}

The photodetachment dynamics probed at $625 \mathrm{~nm}$ dramatically shows the appearance and rapid disappearance of a new species in addition to the wet and solvated electron. This species is also clearly seen to the blue of $625 \mathrm{~nm}$ and a careful inspection of the data shows a small residual component at 850 but none at $1000 \mathrm{~nm}$. The kinetic behavior indicates that the species is either the electron or $\left(\mathrm{I}^{-}\right)^{*}$. The iodine atom is discounted because it does not absorb in the visible [22]. It is well established that the excited state of the iodide anion produced upon excitation corresponds to the CTTS state, where the electron is trapped in the well due to the polarization of the water molecules around the original anion.

To help further confirm the identity of this short lived component, experiments were done on $\mathrm{Br}^{-}$. A similar precursor to the wet electron is seen at 450 $\mathrm{nm}$ for the bromide ion. The observed blue-shift in the absorption spectra of $\mathrm{Br}^{-}$compared with $\mathrm{I}^{-}$was expected due to the change in ionic radius. The ionic radius for the iodide anion is much larger than for the bromide ion, see table 1 . If we think of the CTTS energy levels as that of a particle in a box, the corresponding increase in box size will result in a decrease in the separation of the energy levels. There-

Table 1

\begin{tabular}{llll} 
& $r_{\text {model }}{ }^{\mathrm{a})}(\AA)$ & CTTS $(\mathrm{eV})$ & IP gas $(\mathrm{eV})$ \\
\hline $\mathrm{OH}^{-}$ & 1.76 & 6.6 & 2.17 \\
$\mathrm{Cl}^{-}$ & 2.35 & 6.8 & 3.83 \\
$\mathrm{Br}^{-}$ & 2.52 & 6.2 & 3.55 \\
$\mathrm{I}^{-}$ & 2.75 & 5.4 & 3.24
\end{tabular}

a) The values are taken from ref. [12]. The radii obtained are about $25-30 \%$ larger than the crystallographic radius. 
fore, we expect the electron trapped in the CTTS well to occur at lower energies for the larger iodide anion. This size effect is also consistent with the observed trend in the CTTS absorption maxima as a function of ion size. However, at some point no more bound states will exist in the well and only bound to continuum transitions will be observed.

In fig. 4 the excited state absorption spectra of the CTTS state is shown for $\mathrm{I}^{-}$. The spectra has a clear maxima in the neighborhood of $600 \mathrm{~nm}$. Furthermore, since the signal strength is comparable to the solvated electron and the yield of the solvated electron from the CTTS state is high the absolute absorption coefficient must be large, on the order of $10000 \mathrm{M}^{-1} \mathrm{~cm}^{-1}$. The large absorption coefficient and the shape suggest that the transition is between two bound states; however the maximum could be due to a density of states effect in a bound to continuum transition.

The measured formation time of the wet electron $300 \mathrm{fs}$ represents the radiationless decay rate of the trapped electron into the wet electron. Since the experimentally known quantum yield of solvated electrons produced upon irradiation of $\mathrm{I}_{\mathrm{aq}}^{-}$is less than one $[5,6,23]$, recombination of the trapped electron with the parent species is probably significant. Geminate recombination of the solvated electron would probably not account for the observed quantum yields of $25-30 \%$. We hypothesize that the rate-limiting step in the formation of the wet electron is most likely a nonadiabatic electron transfer process [2426]. The electron must go from a potential surface

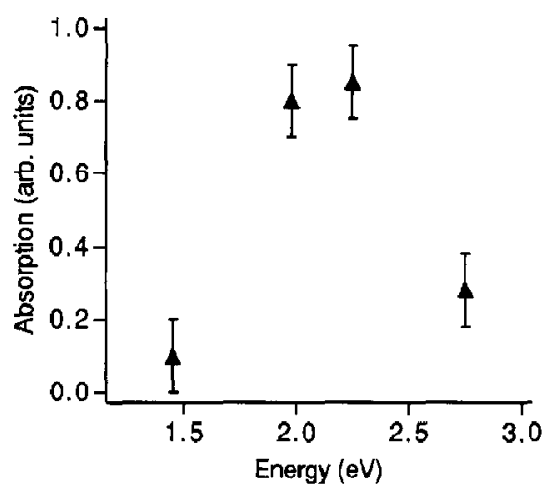

Fig. 4. The absorption spectra of the trapped, i.e. CTTS, electron (triangles).

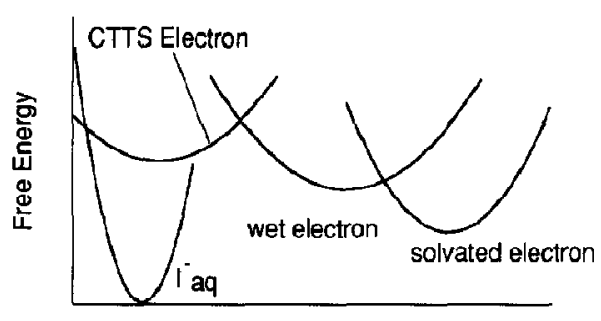

Solvent Coordinates

Fig. 5. A simplied free energy diagram depicting the dynamics of electron photodetachment and solvation. See text for details.

corresponding to the CTTS well to the surface corresponding to the wet electron. A simplified free energy diagram for the process of electron detachment and solvation is shown in fig. 5 . It should be noted that the solvent coordinate (s) involved with the formation of the wet electron are most likely different from those involved in the wet to solvated electron transition. The UV pump pulse causes a vertical transition of the electron to the trapped electron potential surface. The lack of continuous spectral shifts in our experiments and the results of simulations suggest that adiabatic events on these surfaces are very fast $[8,14,15]$. Therefore both the formation and decay of the wet electron can be viewed as surface crossings. However we do recognize that the electronic surfaces could couple strongly and form a single ground state surface, i.e. an adiabatic surface, for the electron to move on. In this case a small barrier could be present and the crossing of this barrier could be the rate-limiting step in the formation of the wet electron. Clearly the situation is complex and more investigation is warranted.

\subsection{Geminate recombination dynamics of the solvated electron}

In a previous report, the geminate recombination dynamics of a solvated electron photodetached from an aqueous chloride and hydroxide anion were reported [7]. In contrast to the geminate recombination dynamics observed for the $\mathrm{OH}$ and $\mathrm{Cl}$ systems, there is no fast component ( $<5$ ps) seen for the I atom solvated electron recombination (fig. 6). In table 2, the fraction of solvated electrons that escape capture (after $70 \mathrm{ps}$ ) is listed. The increase in 


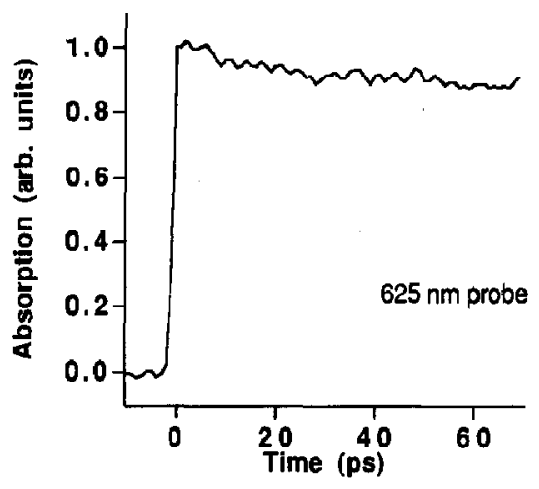

Fig. 6. Geminate recombination dynamics of the solvated electron originating from the iodide ion probed at $625 \mathrm{~nm}$.

Table 2

\begin{tabular}{ll}
\hline Halide & Escape yield a) $(\%)$ \\
\hline $\mathrm{OH}^{-}$ & $70 \pm 3$ \\
$\mathrm{Cl}^{-}$ & $80 \pm 3$ \\
$\mathrm{I}^{-}$ & $89 \pm 3$
\end{tabular}

a) Experimental escape yields measured after 70 ps.

escape yield (and decreased rate of escape) for the larger halides suggest a larger initial electron-atom separation. This is consistent with trends predicted by simple models of the CTTS state. As the ionic size increases the mean radius of the excited states increase rapidly thus leading to larger separation distances. Previously total quantum yields were the only method of obtaining information concerning the dynamics of the CTTS state. These experiments recorded the solvated electron quantum yield as a function of scavenger concentration and temperature $[5,23]$. Our femtosecond time resolved measurements represent a significant advance because they allow for the details of the ultrafast dynamics to be revealed for the first time.

\section{Summary and conclusions}

We have performed femtosecond time-resolved studies of electron photodetachment from simple halide ions in solution. In this Letter we have focused our discussion on the most carefully studied CTTS species, the aqueous iodide ion. The solvation dynamics of the electron photodetached from the iodide ion are qualitatively very different at early times from an electron produced by photoionization of a neutral (i.e. a water molecule). The electron photodetached from a halide ion originates not as a quasifree electron but as an electron trapped in a well formed by the orientational polarization of the water molecules around the once solvated ion. This is the CTTS state. The absorption spectra and dynamics of the trapped electron, i.e. the electron in the photodetached CTTS state, have been measured. It is a precursor of the wet electron. It has a strong absorption spectra centered in the visible around $600 \mathrm{~nm}$ which is very different from either the wet electron or the solvated electron. Similar effects are seen in the $\mathrm{Br}$ anion, where the trapped electron absorption is shifted to the blue, as expected.

The dynamics and absorption spectra of the wet electron have also been measured. The observed formation time of the wet electron is $300 \mathrm{fs}$, about $50 \%$ longer than the value for neat water. The physical mechanism leading to the formation of the wet electron is most likely a nonadiabatic electron transfer process. The electron must go from a potential surface corresponding to the CTTS well to the surface corresponding to the wet electron. The transition of the wet electron into the solvated electron appears to be two-state in character. This was shown by the location of an isosbestic point in the solvation dynamics of the electron. The lifetime of the wet electron was not different from the neat water case. In addition the absorption spectra of the wet electron was not distinctly different than the neat water case. This suggests that the previously proposed hypothesis that the wet electron is really an excited state of the solvated electron is still valid. Further investigation is underway to more clearly elucidate the physical mechanisms involved in the dynamics of photodetachment from simple aqueous anions.

\section{Acknowledgement}

We gratefully acknowledge the financial support of the National Science Foundation, the Air Force Office of Scientific Research and the Petroleum Re- 
search Fund administered by the American Chemical Society.

\section{References}

[1] L.I. Grossweiner and M.S. Matheson, J. Phys. Chem. 61 (1957) 1089.

[2] M.S. Matheson, W.A. Mulac and J. Rabani, J. Phys. Chem. 67 (1963) 261.

[3] G.V. Marr, Photoionization processes in gases (Academic Press, New York, 1967).

[4] J. Jortner, M. Ottolenghi and G. Stein, J. Phys. Chem. 66 (1962) 2029, 2037.

[5] F.S. Dainton and S.R. Logan, Proc. Roy. Soc. A 287 (1965) 281.

[6] M.J. Blandamer and M.F. Fox, Chem. Rev. 70 (1970) 59, and references therein.

[7] F.H. Long, H. Lu and K.B. Eisenthal, J. Chem. Phys. 91 (1989) 4193

[8] F.H. Long, H. Lu and K,B. Eisenthal, Phys. Rev, Letters, submitted for publication.

[9]D. Eisenberg and W, Kauzmann, The structure and properties of water (Oxford Univ. Press, Oxford, 1969).

[10] F. Platzman and J. Frank, L. Farkus memorial volume (Cooperative Printing Press, Haifa, 1952).

[ 11 ] L.D. Landau, Physik. Z. Sowjetunion 3 (1933) 664.
[12] G. Stein and A. Treinin, Trans. Faraday Soc. 55 (1956) 1086.

[13] M. Smith and M.C.R. Symons, Discussions Faraday Soc. 24 (1957) 206.

[14] P.J. Rossky and J. Schnitker, J. Phys. Chem. 92 (1988) 4277.

[15] R,B. Barnett, U. Landman and A. Nitzan J. Chem. Phys. 90 (1989) 4413.

[16] D.F. Coker and B.J. Berne, in: Excess electrons in disordered media, eds. J.-P. Jay-Gerin and C. Ferradini (CRC Press, Boca Raton).

[17] J.M. Wiesenfeld and E.P. Ippen, Chem. Phys. Letters 73 (1980) 47.

[18] A. Migus, Y. Gaudel, J.L. Martin and A. Antonetti, Phys. Rev. Letters 58 (1987) 1559.

[19] H. Lu, F.H. Long, R.M. Bowman and K.B. Eisenthal, J. Phys. Chem. 92 (1989) 27.

[20] F.H. Long, H. Lu and K.B. Eisenthal, Chem. Phys. Letters $160(1989) 464$.

[21] B.D. Micheal, E.J. Hart and K.H. Schmidt, J. Phys. Chem. 75 (1971) 2798.

[22] A. Siegmann, Lasers (University Science Books, Mill Valley, 1986).

[23] J. Jortner, M. Ottolenghi and G. Stein, J. Phys. Chem. 68 (1964) 247.

[24] R.R. Dogonadze in: Reactions of molecules on electrodes, ed. N.S. Hush (Wiley, New York, 1971) ch. 3.

[25] R.A. Marcus and N. Sutin, Biochim. Biophys. Acta 811 (1985) 265

[26] H. Frauenfelder and P.G. Wolynes, Science 229 (1985) 337. 